\title{
The effect of QiangGuYin on osteoporosis through the AKT/mTOR/autophagy signaling pathway mediated by CKIP-1
}

\author{
Yifeng Yuan ${ }^{1,{ }^{*}}$, Jiangang Sun ${ }^{1,{ }^{*}}$, Hang Zhou ${ }^{1,{ }^{*}}$, Shen Wang ${ }^{1,{ }^{*}}$, Caijian He ${ }^{1, *}$, Tianpeng Chen ${ }^{1}$, \\ Mouhao Fang ${ }^{1}$, Shaohua $\mathrm{Li}^{1}$, Shifa Kang ${ }^{1}$, Xiaosheng Huang ${ }^{1}$, Binbin Tang ${ }^{2}$, Bocheng Liang ${ }^{2}$, \\ Yingdelong $\mathrm{MaO}^{2}$, Jianyou $\mathrm{Li}^{3}$, Xiaolin $\mathrm{Shi}^{2, \&}$, Kang $\mathrm{Liu}^{2, \&}$ \\ ${ }^{1}$ The Second Clinical Medical College, Zhejiang Chinese Medical University, Hangzhou, China \\ ${ }^{2}$ Department of Osteology, The Second Affiliated Hospital of Zhejiang Chinese Medical University, Hangzhou, China \\ ${ }^{3}$ Department of Orthopedics, Huzhou Central Hospital, Huzhou, China \\ *Equal contribution
}

Correspondence to: Xiaolin Shi, Kang Liu; email:xlshi-2002@163.com, liukang1982@163.com

Keywords: osteoporosis, CKIP-1, AKT/mTOR signaling pathway, apoptosis, QiangGuYin

Received: July 1, $2021 \quad$ Accepted: November 22, $2021 \quad$ Published: January 24, 2022

Copyright: (C) 2022 Yuan et al. This is an open access article distributed under the terms of the Creative Commons Attribution License (CC BY 3.0), which permits unrestricted use, distribution, and reproduction in any medium, provided the original author and source are credited.

\section{ABSTRACT}

Osteoporosis is a systemic bone disease characterized by decreased bone mass and deterioration of bone microstructure, which leads to increased bone fragility and increased risk of fractures. Casein kinase 2 interacting protein 1 (CKIP-1, also known as PLEKHO1) is involved in the biological process of bone formation, differentiation and apoptosis, and is a negative regulator of bone formation. QiangGuYin (QGY) is a famous TCM formula that has been widely used in China for the clinical treatment of postmenopausal osteoporosis for decades, but the effect in regulating CKIP-1 on osteoporosis is not fully understood. This study aimed to explore the potential mechanism of CKIP-1 participating in autophagy in bone cells through the AKT/mTOR signaling pathway and the regulatory effect of QGY. The results in vivo showed that QGY treatment can significantly improve the bone quality of osteoporotic rats, down-regulate the expression of CKIP-1, LC3II/I and RANKL, and up-regulated the expression of p62, p-AKT/AKT, p-mTOR/mTOR, RUNX2 and OPG. It is worth noting that the results in vitro confirmed that CKIP-1 interacts with AKT. By up-regulating the expression of Atg5 and downregulating the p62, the level of LC3 (autophagosome) is increased, and the cells osteogenesis and differentiation are inhibited. QGY inhibits the combination of CKIP-1 and AKT in osteoblasts, activates the AKT/mTOR signaling pathway, inhibits autophagy, and promotes cell differentiation, thereby exerting an antiosteoporosis effect. Therefore, QGY targeting CKIP-1 to regulate the AKT/mTOR-autophagy signaling pathway may represent a promising drug candidate for the treatment of osteoporosis.

\section{INTRODUCTION}

Osteoporosis (OP) is a systemic multifactorial skeletal disorder characterized by decreased bone mass, deterioration of bone microarchitecture and increased bone fragility, resulting in a tendency of fracture and leading to possible lifelong disability or death $[1,2]$. With the expanding ageing population, the prevalence of $\mathrm{OP}$ is increasing, and it has become an important public health concern in many countries [3]. Researches have shown that the imbalance between osteoblast-mediated bone formation and osteoclast-mediated bone resorption, which leads to OP [4]. Among them, the imbalance in the number and activity of osteoblasts and osteoclasts plays a major role [5]. Considering that the adverse reactions of the present used drugs in clinical with accurate efficacy are also obvious, the development of possible novel therapeutic strategies has become of real importance. 
CKIP-1 mediates cell growth, differentiation, apoptosis, cytoskeleton [6, 7], and bone formation, and has been found to be a promising target for OP therapy. Studies have shown that CKIP-1 knockout (KO) mice increase bone density and bone mass with age, and the activity of osteoblasts is significantly higher than those of wildtype mice [8], while the small interfering RNA of CKIP (siRNA) significantly promoted bone formation in vivo and in vitro [9]. Autophagy is the main catabolic process for eukaryotic cells to degrade and recycle damaged macromolecules or organelles. As a cell survival pathway, it plays a vital role in maintaining bone homeostasis, and the changes in this pathway are to some extent associated with osteoporosis [10-13].

Herbal medicines, especially traditional Chinese medicines (TCMs), which are based on natural compounds, have been clinically used in Asian countries for more than 2,500 years, for the treatment of various diseases including osteoporosis [14-17]. For example, Bu-Shen-Ning-Xin decoction was reported to suppress osteoclastogenesis by regulating RANKL/OPG ratio and protected mice from osteoporosis [17]. Another TCM formula, Fufang Zhenshu Tiaozhi (FTZ), also processed anti-osteoporotic activities against aginginduced osteoporosis in mice [14]. Wang et al. reported that another TCM formula Gushukang granules protected mice from osteoporosis by inhibiting osteoclastogenesis and stimulating osteoblastogenesis [16]. Based on the widely published TCM-related studies, few adverse effects were reported. Thus, growing attentions were attracted for the clinical usage and research application of TCMs. QiangGuYin (QGY) is a famous TCM formula that has been widely used in China for the clinical treatment of postmenopausal osteoporosis for almost 20 years [18-20]. In 2017, we published our multi-center clinical trial involving 240 participants. Based on the results of our study, 1-Year treatment with QGY effectively and safely increased Bone Mineral Density (BMD) and reversed osteoporotic bone turn over [20]. The underlying mechanism, however, remained to be uncovered. Therefore, this study tried to explore the potential mechanism of CKIP1 involved in autophagy in bone cells through the AKT/mTOR signaling pathway and the regulation of QGY.

\section{MATERIALS AND METHODS}

\section{Experimental animal}

Adult female Sprague-Dawley (SD) rats were purchased from the Experimental Animal Center of Zhejiang University of Traditional Chinese Medicine and were fed under environmental conditions without specific pathogens (temperature $20-25^{\circ} \mathrm{C}$, relative humidity 55-
$65 \%$, light/dark cycle $12 \mathrm{~h}$ ) And maintain free access to water and food. All experimental procedures follow the Guidelines for the Care and Use of NIH Laboratory Animals (NIH Publication No. 80-23, revised in 1978) and have been approved by the Animal Care and Use Committee of Zhejiang University of Traditional Chinese Medicine (Approval Number: 20200622-09).

\section{Preparation of QGY medicines and pharmacy- containing serums}

QGY (No. Z20195155) is produced by the Pharmaceutical Preparation Center of the Second Affiliated Hospital of Zhejiang University of Traditional Chinese Medicine. Each bottle of QGY contains $22.2 \mathrm{~mL}$ concentrated soup, which were composed of $245 \mathrm{~g}$ crude drugs, including honeysuckle stem 25 g, Cornu Cervi Degelatinatum 20 g, Caulis Spatholobi 25 g, Nidus Vespae 20 g, Gentiana macrophylla 15 g, Radix Sileris $15 \mathrm{~g}$, cinnamon $10 \mathrm{~g}$, Ligusticum wallichii $20 \mathrm{~g}$, Eucommia ulmoides $15 \mathrm{~g}$, Astragalus membranaceus 30g, Rhizoma Drynariae 20 $\mathrm{g}$, and Dipsacus asperoides $30 \mathrm{~g}$. The drug-containing serum was prepared as follows: 40 male SD rats weighed 280-300 g were randomly divided into control or drug groups. In drug group, rats were intragastrically administered QGY at a dosage of $10 \mathrm{~mL} / \mathrm{kg}$ twice a day. In control group, PBS was administered instead of QGY. After 3 days, rats were euthanized and blood was isolated by eyeball extirpating. Then, serum from drug group (QGY-serum) and control group (control-serum) were isolated and used in vitro experiments.

\section{Animal osteoporosis model}

After 12-week-old female SD rats (body weight 280$300 \mathrm{~g}$ ) were adaptively fed for 3 days, 5 rats were subjected to a bilateral laparotomy group (Sham), and another 30 rats were subjected to bilateral ovariectomy according to the previous experimental method (OVX) [21]. OVX rats were randomly divided into 6 groups $(n=5)$ : vehicle group $(\mathrm{OVX}+\mathrm{PBS})$, low-dose QGY group (OVX+L-QGY, $2.5 \mathrm{~mL} / \mathrm{kg}$ /day), medium-dose QGY group (OVX+M-QGY, $5 \mathrm{~mL} / \mathrm{kg} /$ day), high-dose QGY group (OVX+H-QGY, $10 \mathrm{~mL} / \mathrm{kg} /$ day), autophagy inhibitor group (OVX+3-MA), high-dose QGY + autophagy inhibitor group (OVX+H-QGY+3-MA). All drugs were treated orally for 6 weeks. After completing the experiment, samples of the right femur of each group of rats were taken for histological staining detection and micro-CT scan.

\section{Histomorphometric analysis}

A micro-CT imaging system (Skyscan 1176, Belgium) was used for morphological analysis of trabecular bone. 
The left femur sample was scanned at a resolution of $14.8 \mu \mathrm{m}$. Select the trabecular bone region of interest to quantify BMC. By measuring bone trabecular volume fraction $(\mathrm{BV} / \mathrm{TV}, \%)$, bone density $(\mathrm{BMC} / \mathrm{TV})$, bone trabecular number (Tb.N) and bone trabecular spacing (Tb.Sp, $\mathrm{mm}$ ) and other related parameters, Reflects the internal microstructure of the femur.

The femur sample was fixed in $4 \%$ paraformaldehyde, then the sample was embedded in paraffin and cut into 4 $\mu \mathrm{m}$ thick sections. Use hematoxylin-eosin (H\&E) staining kit to observe the cell morphology of trabecular bone. In addition, according to the manufacturer's instructions, slices were fixed in TRAP fixative solution at $4^{\circ} \mathrm{C}$ for $3 \mathrm{~min}$, then incubated in TRAP working solution at $37^{\circ} \mathrm{C}$ in the dark for $60 \mathrm{~min}$, stained with hematoxylin solution for 3-5 min, and then subjected to microscopic examination to confirm the bone cell differentiation.

\section{Bone cell culture and differentiation}

MC3T3-E1 osteoblasts and RAW264.7 cells were purchased from Cell Bank of Chinese Academy of Sciences (Shanghai, China). MC3T3-E1 osteoblasts were cultured in $\alpha$-minimum essential medium (Gibco; Thermo Fisher Scientific, Inc., Waltham, MA, USA) with $10 \%$ fetal bovine serum (Gibco) and $1 \%$ penicillinstreptomycin. Cells were maintained in medium with ascorbic acid $(50 \mu \mathrm{g} / \mathrm{mL})$ and $\beta$-glycerol phosphatase $(10 \mathrm{mM})$ for osteogenic differentiation for 21 days. In addition, RAW264.7 cells were cultured using a-MEM $(10 \% \mathrm{FBS}, 1 \% \mathrm{P} / \mathrm{S})$ and maintained at $37^{\circ} \mathrm{C}$ with $5 \%$ $\mathrm{CO}_{2}$ overnight, and were stimulated with M-CSF $(20 \mathrm{ng} / \mathrm{mL})$ and RANKL $(50 \mathrm{ng} / \mathrm{mL})$ in a-MEM to induce osteoclasts formation $\left(1 \times 10^{3}\right.$ cells/well in 96 well plates).

\section{Western blotting analysis}

Lyse the tissue or cells in pre-cooled lysate $(50 \mathrm{mM}$ Tris-Cl, pH 7.4, 1 mM EDTA pH 8.0, $250 \mathrm{mM} \mathrm{NaCl}$, and $1 \%$ Triton-X) for 30 minutes, and centrifuge at $12000 \mathrm{rpm}$ at $4^{\circ} \mathrm{C}$ for 20 minutes. The supernatant was collected and the total protein concentration was determined by BCA protein analysis kit (Beyotime, Shanghai, China). The same amount of protein $(40 \mu \mathrm{g})$ was separated by $10 \%$ SDS-PAGE and transferred to PVDF membrane (Bio-Rad). The membrane was blocked with $4 \%$ skimmed milk for $2 \mathrm{~h}$ and incubated overnight at $4^{\circ} \mathrm{C}$ with the following primary antibodies: CKIP-1 (1:1000, Santa Cruz Biotechnology, SC-376355), LC3II/I (1:1000, Abcam, ab128065), p62 (1:1000, Abcam, ab91526), Atg5 (1:1000, Proteintech, 10181-2-AP), AKT (1:1000, Cell Signaling Technology, 9272), phospho-AKT
(1:1000, CST, 4060), mTOR (1:1000, Cell Signaling Technology, 2983), phospho-mTOR (1:1000, Cell Signaling Technology, 2971), RUNX2 (1:1000, Abcam, ab236639), RANKL (1:1000, Santa Cruz Biotechnology, SC-377079), OPG (1:1000, Santa Cruz Biotechnology, SC-390518) and GAPDH (1:5000, Santa Cruz Biotechnology, United States, sc25778). The membrane was incubated with HRPconjugated antibody (Wuhan Boster Bioengineering Co., Ltd.) for $1 \mathrm{~h}$, and then the enhanced ECL chemiluminescence reagent (Bio-Rad) and GelDoc imaging system (BioRad) were used to quantify immunoreactive bands.

\section{Construction of CKIP-1 expression lentivirus and knockdown shRNA}

CKIP-1 expression lentivirus and knockdown shRNA were constructed using standard methods. In detail, Taq Plus DNA Polymerase (ET105-02-01, TIANGEN), dNTP (CD117, TIANGEN), T4 DNA Ligase (FL10102, TRANS), Xho I (1094A, TAKARA), Not I (1166A, TAKARA), DL15000 DNA Maker (MD110, TIANGEN), DL2000 DNA Maker (MD114, TIANGEN) were used according to the manufactures' instructions. MC3T3-E1 cells and RAW 264.7 cells were then transfected with lentivirus and shRNA using polybrene (Sigma) for $24 \mathrm{~h}$ and passaged for following experiments.

\section{Co-IP detection}

Total cellular proteins of osteoblasts from different groups were extracted. Extracted proteins (500 $\mu \mathrm{g} /$ sample) were pretreated with $30 \mu \mathrm{L}$ Agarose Protein $\mathrm{A}+\mathrm{G}$ beads (CST, \#9863 and 37478) for $2 \mathrm{~h}$ at $4^{\circ} \mathrm{C}$. Then the beads were removed by centrifuge. After that, $3 \mu \mathrm{g}$ HA-Tag rabbit monoclonal antibody (IP-HA, $1: 100$, CST, \#3724) or IgG (IP-IgG, 1:100, CST, \#3900) was added into samples and incubated overnight at $4^{\circ} \mathrm{C}$. Another $30 \mu \mathrm{l}$ Agarose Protein $\mathrm{A}+\mathrm{G}$ beads $(50 \%)$ were added into samples and incubated for $6 \mathrm{~h}$ at $4^{\circ} \mathrm{C}$. After centrifuge, the sediment was isolated and resuspended with $30 \mu \mathrm{L}$ loading buffer. Then, the proteins were measured by western blot as described above.

\section{LC3 immunofluorescence assay}

The cells were fixed in $4 \%$ paraformaldehyde (Solarbio, Beijing, China) and blocked in 5\% BSA for $1 \mathrm{~h}$, and then incubated with rabbit monoclonal anti-LC3B antibody (Cell Signaling Technology, Danvers, MA, USA) overnight and with Alexa Fluor The 488-conjugated secondary antibody was incubated for $1 \mathrm{~h}$. Finally, it was 
counterstained with 4',6-dimidyl-2-phenylindole dye (DAPI) (Cell Signaling Technology, Danvers, MA, USA), and performed on a confocal laser scanning microscope (Leica, SP8, Germany) Capture images on.

\section{ELISA analysis}

7 days after osteoblast induction, ELISA was used to measure the protein expression levels of RANKL and OPG according to the manufacturer's instructions.

\section{Quantitative real-time PCR}

Total RNA was removed from the cells using Trizol reagent (Invitrogen, Carlsbad, NM, USA) according to the manufacturer's instructions. cDNA was synthesized with ReverTra Ace qPCR RT Master Mix (Toyobo, Osaka, Japan). Real-time PCR was performed on an ABI 7500 Fast Real-Time PCR System using SYBR Green PCR Master Mix (Applied Biosystems, Foster City, CA, USA). The optimal conditions for thermal cycling are as follows: initial denaturation at $95^{\circ} \mathrm{C}$ for $15 \mathrm{~min}$, followed by denaturation at $95^{\circ} \mathrm{C}$ for $30 \mathrm{~s}$, annealing at $60 \pm 3^{\circ} \mathrm{C}$ for $30 \mathrm{~s}$, and extension at $60^{\circ} \mathrm{C}$ for $30 \mathrm{~s}$ for 40 cycles. The primers used for real-time PCR are as follows: CKIPI (Forward): 5'-GCCGTGAGTCCTGAAGAGAAG-3', (Reverse): 5'-CGAGTAGGGTGGGCAAGATAG-3'; OPG (Forward): 5'-CCTGCCTGGGAAGAAGATCA-3', (Reverse): 5'-TTGTGAAGCTGTGCAGGAAC-3'; RANKL (Forward): 5'-GCACACCTCACCATCAATG CT-3', (Reverse): 5'-GGTACCAAGAGGACAGAGTG ACTTTA-3'; GAPDH (Forward): 5'-ATGGGTGTGA ACCACGAGA-3', (Reverse): 5'-CAGGGATGATGTT CTGGGCA-3'.

\section{Alizarin Red Staining}

The cells were fixed with $4 \%$ paraformaldehyde (Solarbio, Beijing, China) for $10 \mathrm{~min}$ at room temperature. According to the manufacturer's instructions, the cells were stained with Alizarin Red Staining Kit (Beyotime, Shanghai, China) for $60 \mathrm{~min}$ at room temperature. After washing 3 times, photograph the stained cells immediately.

\section{Statistical analysis}

All data were presented as mean \pm standard deviation (mean $\pm \mathrm{SD}$ ). Differences among data from 3 or more groups were analyzed by one-way ANOVA with post hoc Tukey's multiple comparison tests. Analyses were performed using Prism software (v. 8.0; GraphPad, San Diego, CA, USA). $p<0.05$ were recognized as statistically significant.

\section{RESULTS}

\section{QGY improves bone condition in osteoporotic rats}

To determine whether QGY restored bone loss caused by estrogen deficiency, OVX rats were treated with different doses of QGY for 6 weeks. The results of micro-CT three-dimensional imaging showed that compared with the Sham group, the OVX+PBS group had lower bone density and reduced bone trabeculae; QGY and autophagy inhibitor 3-MA treatments could improve bone conditions, and the effective effect of QGY was dose-dependent (Figure 1A). Further analysis of the image parameters found that compared with the Sham group, the BV/TV, BMC/TV, Tb.N index of the OVX+PBS group decreased, and the TB.Sp index increased; QGY treatment significantly increased $\mathrm{BV} / \mathrm{TV}$ and bone density, and decreased $\mathrm{Tb} \mathrm{Sp}$, and is dose-dependent; autophagy inhibitor 3-MA treatment can significantly increase BV/TV and bone density. At the same time, high-dose QGY and 3-MA treatment can significantly improve bone microstructure (Figure 1B).

To further evaluate the influence of QGY on bone structure, $H \& E$ staining was used to determine the thickness and number of bone trabeculae. Compared with the Sham group, the bone marrow cavity in the OVX+PBS group became larger, the bone trabeculae became thinner, and the structure was disordered and loose; QGY and 3-MA treatments thickened the bone trabeculae to varying degrees, the bone marrow cavity became smaller, and the structure was compact (Figure 2A). In order to evaluate the effect of QGY on osteoclasts, TRAP staining was used to determine the differentiation of osteoclasts. TRAP staining showed that the osteoclasts were wine-red, located at the edge of the trabecular bone. Compared with the Sham group, the number of osteoclasts in the OVX+PBS group was significantly increased; QGY and 3-MA treatments significantly reduced the number of osteoclasts (Figure 2B). These results indicate that QGY can improve the bone condition of osteoporotic rats in a dose-dependent manner, possibly by regulating autophagy.

QGY reduces the expression of CKIP-1 in the femoral tissue of osteoporotic rats and mediates autophagy through the AKT/mTOR pathway

In order to study the molecular mechanism of QGY improving osteoporosis, the expression of related proteins in the autophagy pathway mediated by CKIP-1 and AKT/mTOR was evaluated. Western blotting analysis showed that, compared with the Sham group, the expressions of CKIP-1, LC3II/I and RANKL were up-regulated in the femoral tissues of the OVX+PBS group, while the expressions of p62, p-AKT, p-mTOR, 


\section{A}

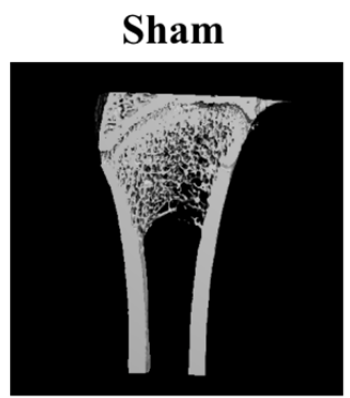

OVX+H-QGY
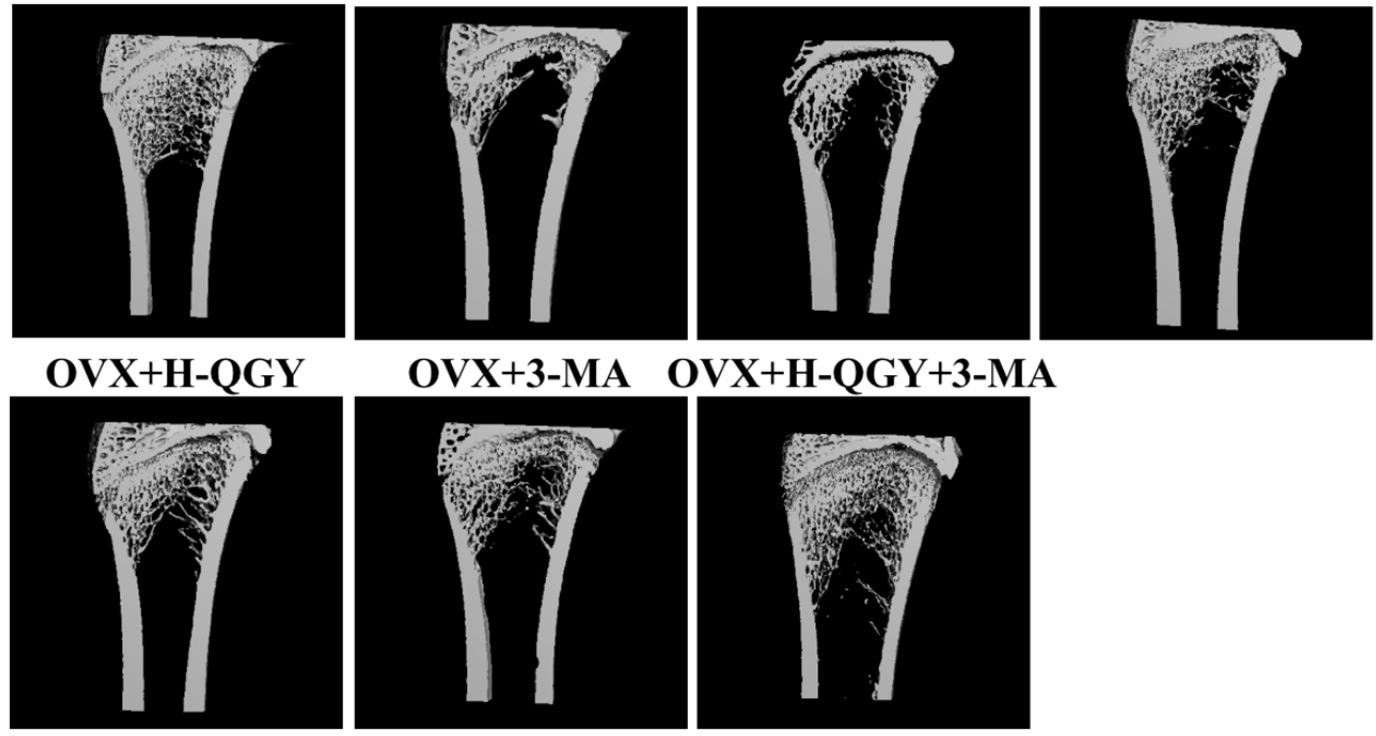

\section{OVX+3-MA OVX+H-QGY+3-MA}
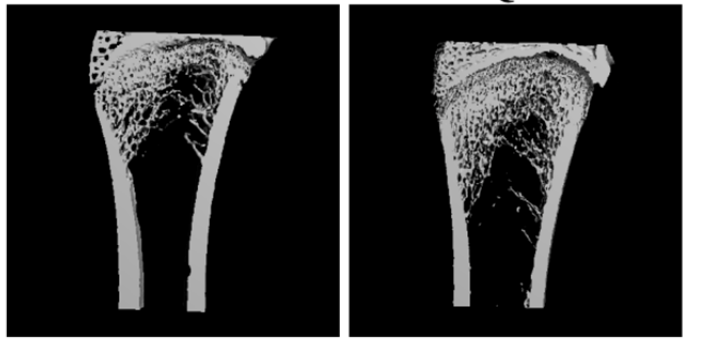

B

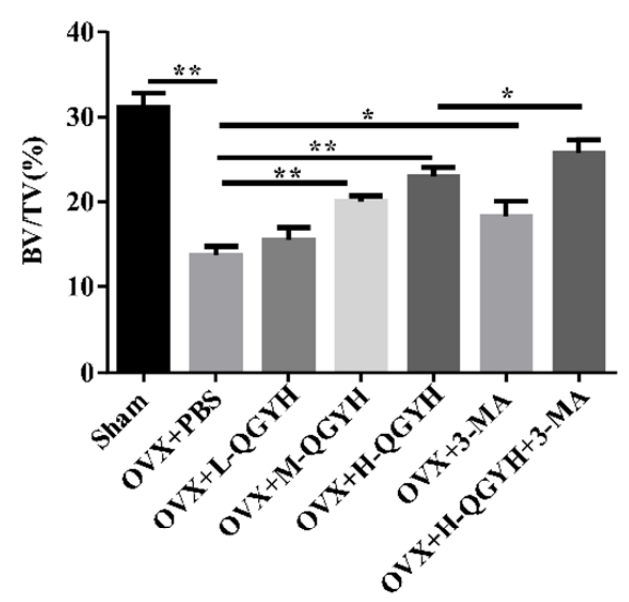

D

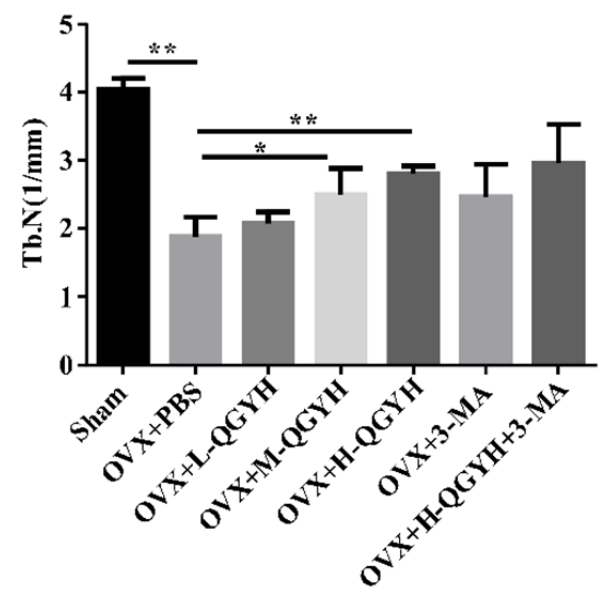

C

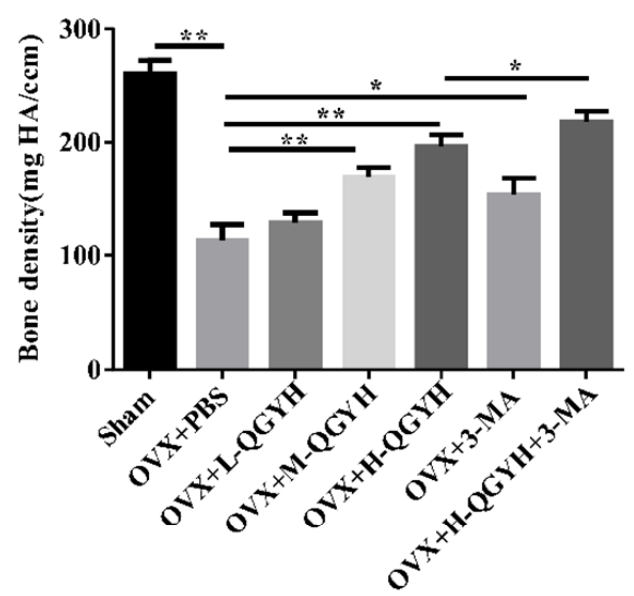

E

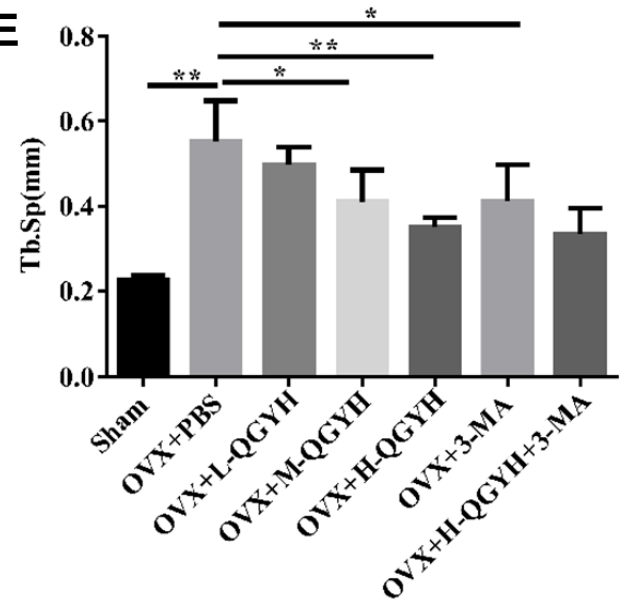

Figure 1. Micro-CT analysis after treating with QGY in animal model. Ovariectomy (OVX) was performed in rats, and then different doses of QGY were given to the rats. (A) Representative figures of three-dimensional micro-CT analysis. (B-E) Quantitative analysis of bone trabecular volume fraction (BV/TV, \%) (B), bone density (BMC/TV) (C), bone trabecular number (Tb.N) (D) and bone trabecular spacing (Tb.Sp, $\mathrm{mm})(\mathrm{E})$ in three-dimensional micro-CT analysis. The data are presented as the means $\pm \mathrm{SD}(\mathrm{n}=5) .{ }^{*} p<0.05,{ }^{* *} p<0.01$. One-way ANOVA followed by Tukey's post hoc test. 
RUNX2, and OPG were down-regulated, suggesting that the increase of autophagy levels inhibit the AKT/mTOR signaling pathway; QGY and 3-MA treatment reverses the expression level of related proteins, and the effect of QGY shows a dosedependent characteristic (Figure 3A, 3B). The gene expression levels of CKIP-1, RANKL and OPG were further checked, and it was found that QGY and 3-MA treatments also reduced the gene expression levels of CKIP-1 and RANKL and promoted the gene expression level of OPG (Figure 3C). It is speculated that QGY may inhibit autophagy, activate the AKT/mTOR pathway, reduce the expression of CKIP-1 and RANKL, and promote the expression of RUNX2 and OPG, thereby improving osteoporosis.

\section{QGY mediates the effect of CKIP-1 on autophagy of osteoblasts and osteoclasts}

In order to study the mechanism of QGY-mediated CKIP-1 in improving osteoporosis, the interaction between CKIP-1 and AKT was first evaluated. The results showed that in osteoblasts, compared with the HA-CKIP-1-OE group, the binding content of CKIP-1 and AKT decreased significantly after QGY treatment, indicating that CKIP-1 and AKT interact, and QGY treatment can reduce its binding capacity; the opposite trend appears in osteoclasts (Figure 4A). These results indicate that there is an interaction between CKIP-1 and AKT, and QGY inhibits the binding of CKIP-1 and AKT in osteoblasts and promotes the binding of CKIP-1 and AKT in osteoclasts.

Further analyze the effect of QGY on autophagy. First, it was determined that QGY could reduce the mRNA level after CKIP-1 overexpression (Figure 4F). In osteoblasts, compared with the control group, the Atg5 protein expression level of the CKIP-1 OE group increased, the p62 protein level decreased (Figure 4B), and the LC3 punctate aggregates (autophagosomes) increased (Figure 4D), indicating that CKIP-1 mediates autophagy activation of osteoblasts. On the contrary, in
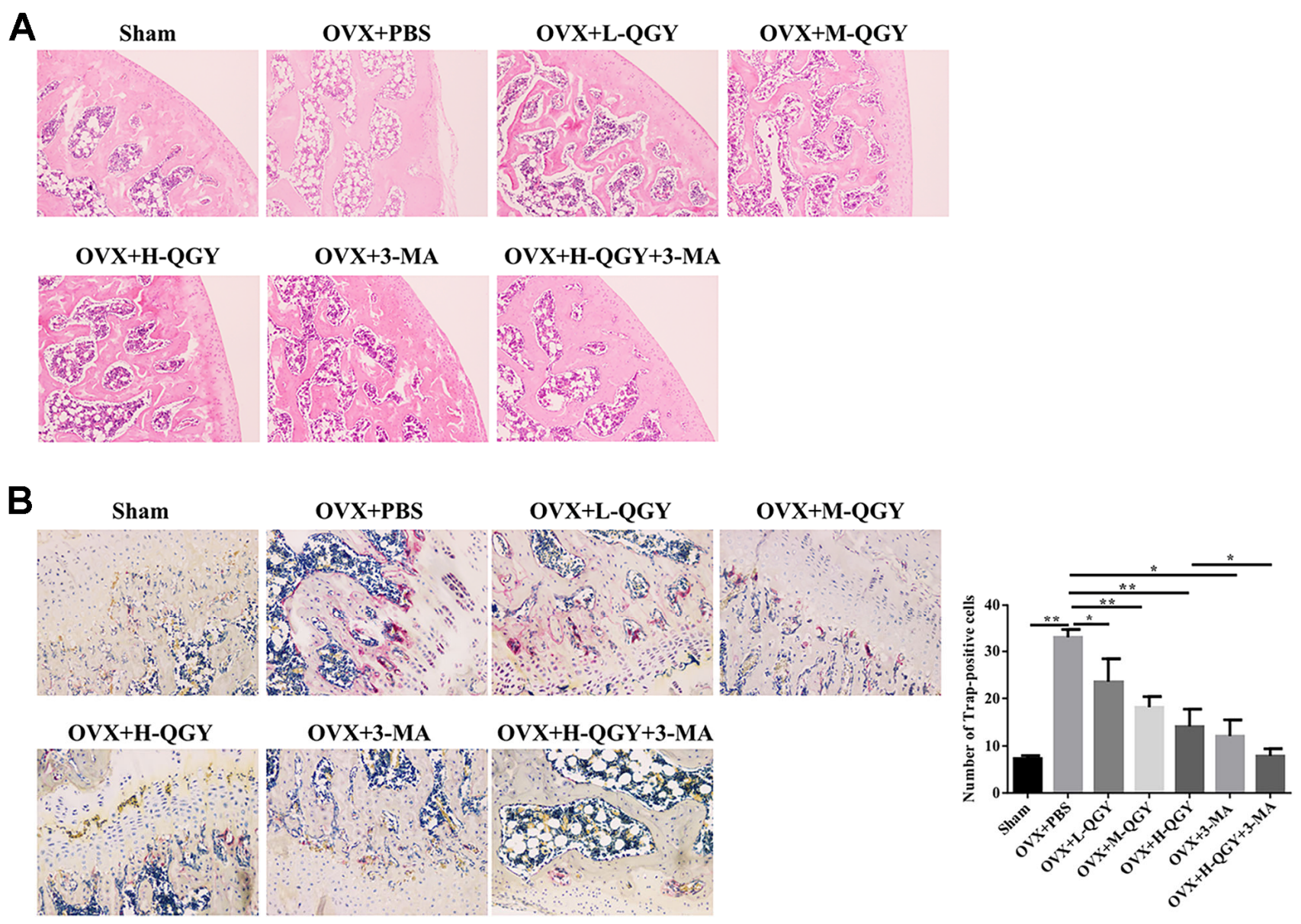

Figure 2. Staining analysis after treating with QGY in animal model. (A) H\&E staining of the trabecular bone. (B) TRAP staining and quantitative analysis of the trabecular bone. The data are presented as the means $\pm S D(n=5) .{ }^{*} p<0.05,{ }^{* *} p<0.01$. One-way ANOVA followed by Tukey's post hoc test. 
osteoclasts, compared with the control group, the Atg5 protein expression level of the CKIP-1 OE group decreased, the p62 protein level increased (Figure 4C), and the LC3 punctate aggregates decreased (Figure 4D). This indicates that CKIP-1 mediated autophagy inhibition, and QGY treatment reversed CKIP-1 mediated osteoblasts and osteoclast autophagy. Next, the regulation of autophagy was tested. In osteoblasts, compared with the CKIP-1 OE infection group, after the addition of autophagy inhibitor 3-MA, the intracellular LC3 II/I level decreased, and the p62 protein expression level increased; after the addition of the autophagy activator rapamycin, LC3 II/I level increased, but p62 protein expression level decreased; after adding QGY treatment, LC3 II/I level decreased, p62 protein expression level increased; autophagy inhibitor 3-MA treatment can increase the autophagy inhibitory effect of QGY on cell autophagy, and autophagy activator rapamycin can also increase the activation of QGY on cell autophagy (Figure 5A). In osteoclasts, this trend is reversed (Figure 5B). These results indicate that QGY inhibits the combination of CKIP-1 and AKT, activates the AKT/mTOR pathway, inhibits autophagy in osteoblasts, thereby improving osteoporosis, which is consistent with the results of in vivo experiments.
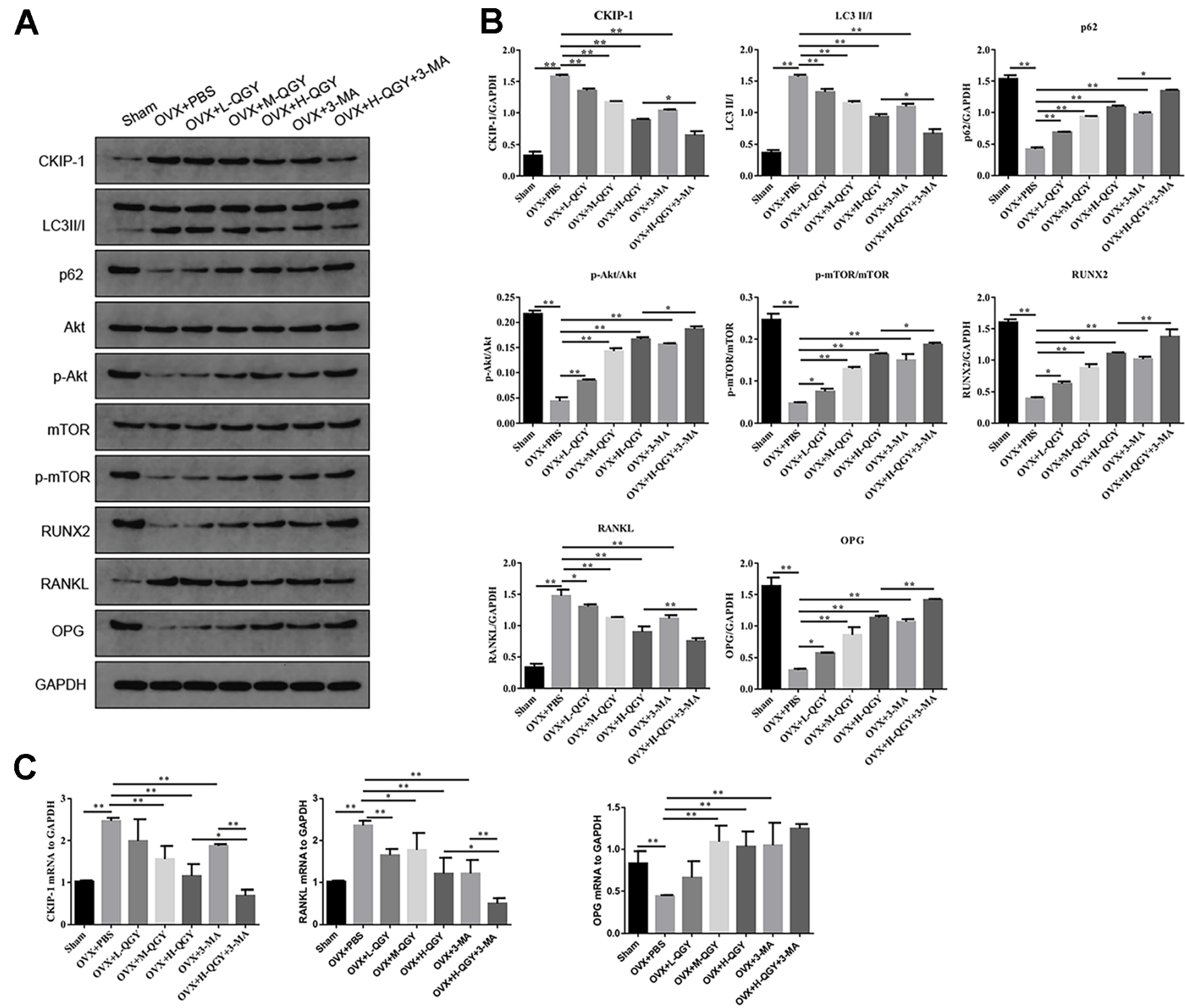

Figure 3. The effect of QGY on the protein expression of femoral tissue in osteoporotic rats. (A, B) Representative figures (A) and quantitative analysis (B) of protein expression of CKIP-1, LC3II/I, p62, p-AKT/AKT, p-mTOR/mTOR, RUNX2, RANKL and OPG. (C) Gene expression levels of CKIP-1, RANKL and OPG. The data are presented as the means \pm SD $(n=5) .{ }^{*} p<0.05,{ }^{* *} p<0.01$. One-way ANOVA followed by Tukey's post hoc test. 
The effect of CKIP-1 mediated autophagy on the differentiation and function of osteoblasts and osteoclasts

The differentiation ability of osteoblasts was evaluated by Alizarin Red staining. The results showed that 3-MA treatment can significantly increase the calcified nodules in osteoblasts infected with CKIP-1 OE, and the osteogenic capacity is enhanced; after rapamycin treatment, the calcified nodules are reduced and the osteogenic capacity is reduced; after QGY treatment, the calcified nodules are obvious, and the osteogenic ability is enhanced; 3-MA can enhance the deposition of
QGY on the calcified nodules of osteoblasts, and rapamycin can inhibit the promotion of QGY on the calcified nodules of osteoblasts (Figure 6A). These results indicate that QGY promotes the differentiation ability of osteoblasts and enhances the osteogenic ability by inhibiting the level of autophagy.

Further detect the content of RANKL and OPG in the supernatant of osteoblasts. The results showed that compared with the control group, the RANKL content in the supernatant of CKIP-1 OE group was significantly increased, and the OPG content was significantly reduced; QGY treatment could reduce
A

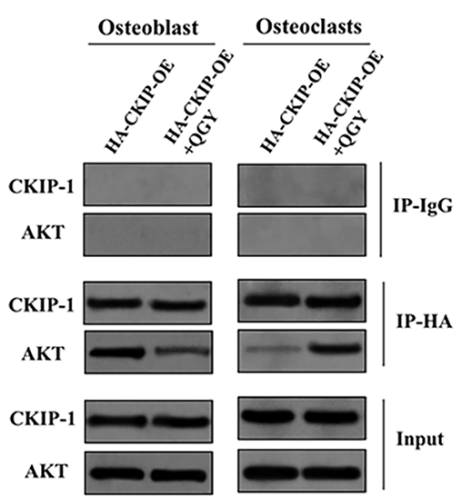

D

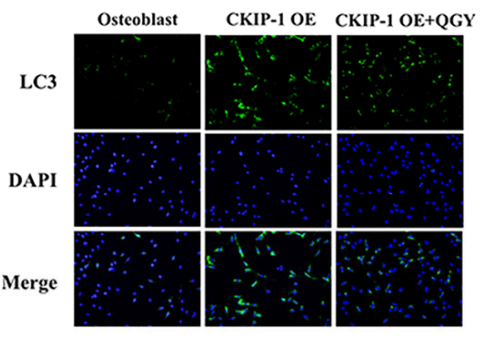

B
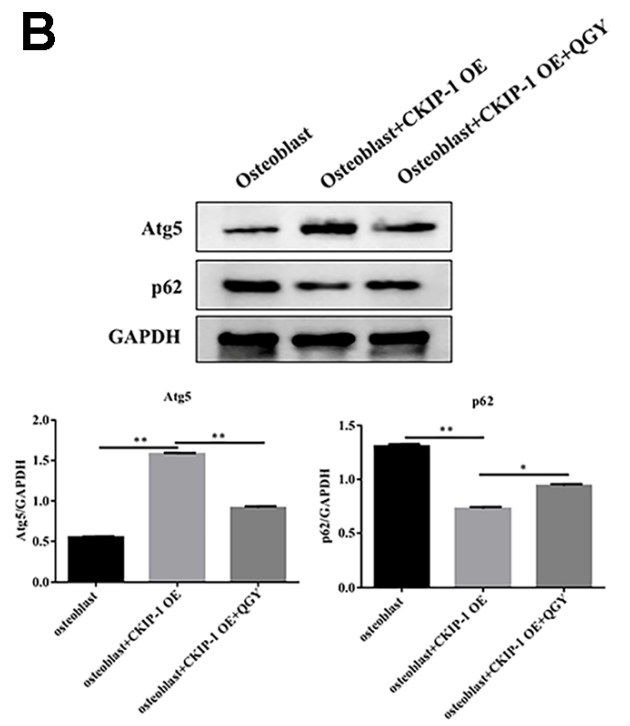

E
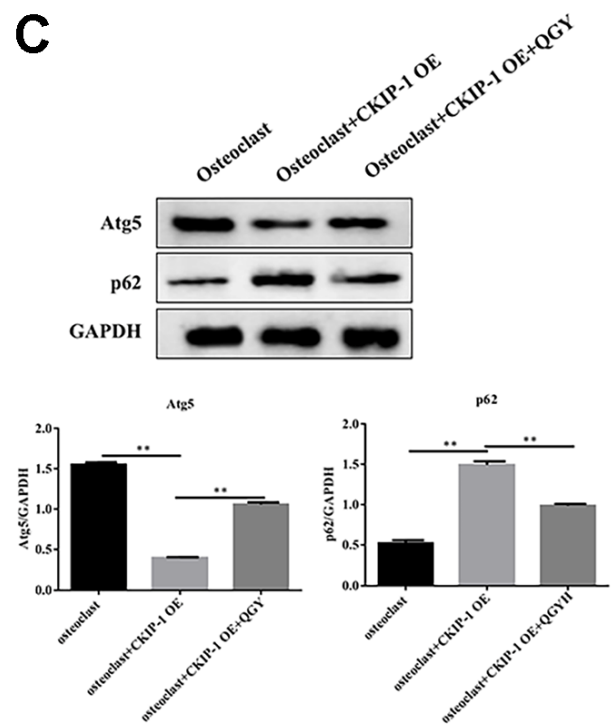

Osteoblast
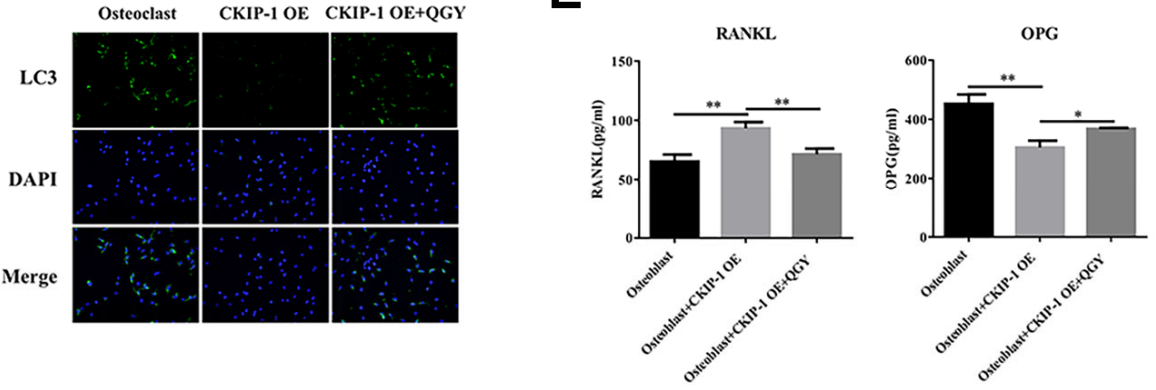

$\mathbf{F}$

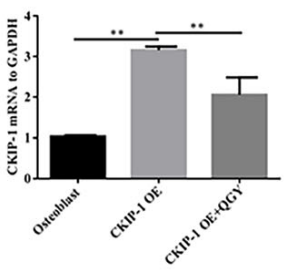

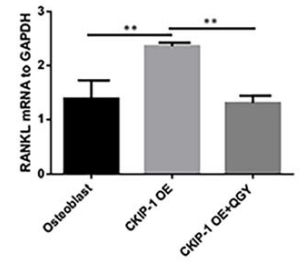

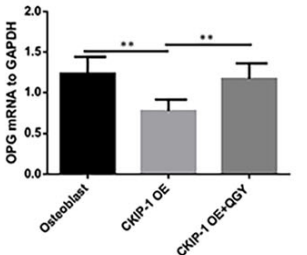

Figure 4. QGY mediates the effect of CKIP-1 on autophagy through the AKT/mTOR pathway. (A) Co-IP assay in the osteoblasts and osteoclasts. (B, C) Representative figures and quantitative analysis of protein expression of Atg5 and p62 in the osteoblasts (B) and osteoclasts (C). (D) LC3 immunofluorescence assay in the osteoblasts and osteoclasts. (E) RANKL and OPG content analysis in the osteoblasts. (F) Gene expression levels of CKIP-1, RANKL and OPG in the osteoblasts. The data are presented as the means \pm SD (B, C, E, F: $n=6 ; \mathbf{D}: n=3)$. ${ }^{*} p<0.05,{ }^{* *} p<0.01$. One-way ANOVA followed by Tukey's post hoc test. 
RANKL content and increased OPG content, indicating that QGY inhibits osteoblast secretion of RANKL and promotes OPG secretion (Figure 4E). Treatment with autophagy inhibitor 3-MA can significantly reduce RANKL secretion and increase OPG secretion in osteoblast, and increase the regulation of QGY on RANKL and OPG secretion; treatment with autophagy inducer rapamycin can increase RANKL secretes and inhibits OPG secretion, and inhibits the regulation of QGY (Figure 6B). Similarly, the mRNA levels of RANKL and OPG showed a consistent trend (Figures $4 \mathrm{~F}, 6 \mathrm{E})$. These results indicate that $\mathrm{QGY}$ inhibits the secretion of RANKL by osteoblasts and promotes the secretion of OPG by regulating autophagy, which is consistent with the results of in vivo experiments.

Western blotting analysis was used to detect the expression level of osteoblast marker RUNX2 protein. The results show that the autophagy inhibitor 3-MA can promote the protein expression of RUNX2 in the osteoblasts CKIP-1 OE group; the autophagy activator rapamycin can inhibit the protein expression of RUNX2; QGY treatment can increase the protein expression of RUNX2. In addition, 3-MA can enhance the promotion of QGY on RUNX2 expression, while rapamycin can block the induction of QGY on RUNX2 expression in osteoblast (Figure 6C). To evaluate the anti-tartrate acid phosphatase (TRACP) activity of osteoclasts. The results showed that treatment with 3-MA can inhibit TRACP activity of osteoclasts infected by CKIP-1 OE; treatment with rapamycin can increase TRACP activity. In addition, QGY treatment can inhibit TRACP activity; 3-MA can enhance the inhibitory effect of QGY on osteoclast TRACP activity, and rapamycin can inhibit the inhibitory effect of QGY (Figure 6D). These results indicate that QGY inhibits TRACP activity through autophagy regulation on osteoclast.

\section{DISCUSSION}

CKIP-1 is a transcription factor that acts as a scaffold linker to mediate interactions with various signals and cellular proteins, and is considered to be an important gene responsible for the development and progression of osteoporosis. Previous studies have shown that CKIP-1 is a regulator of cell viability, apoptosis, cytoskeleton formation and cell differentiation. Recent studies have also confirmed that CKIP-1 is a negative regulator of bone formation, leading to excessive activation of osteoclasts and bone loss. Drugs targeting osteoblasts CKIP-1 can reverse bone formation and prevent osteoporosis [22]. In CKIP-1 KO mice, osteoblast activity and BMD were significantly increased [23]. SiRNA knockout targeting CKIP-1 improved bone formation and osseointegration in a rat
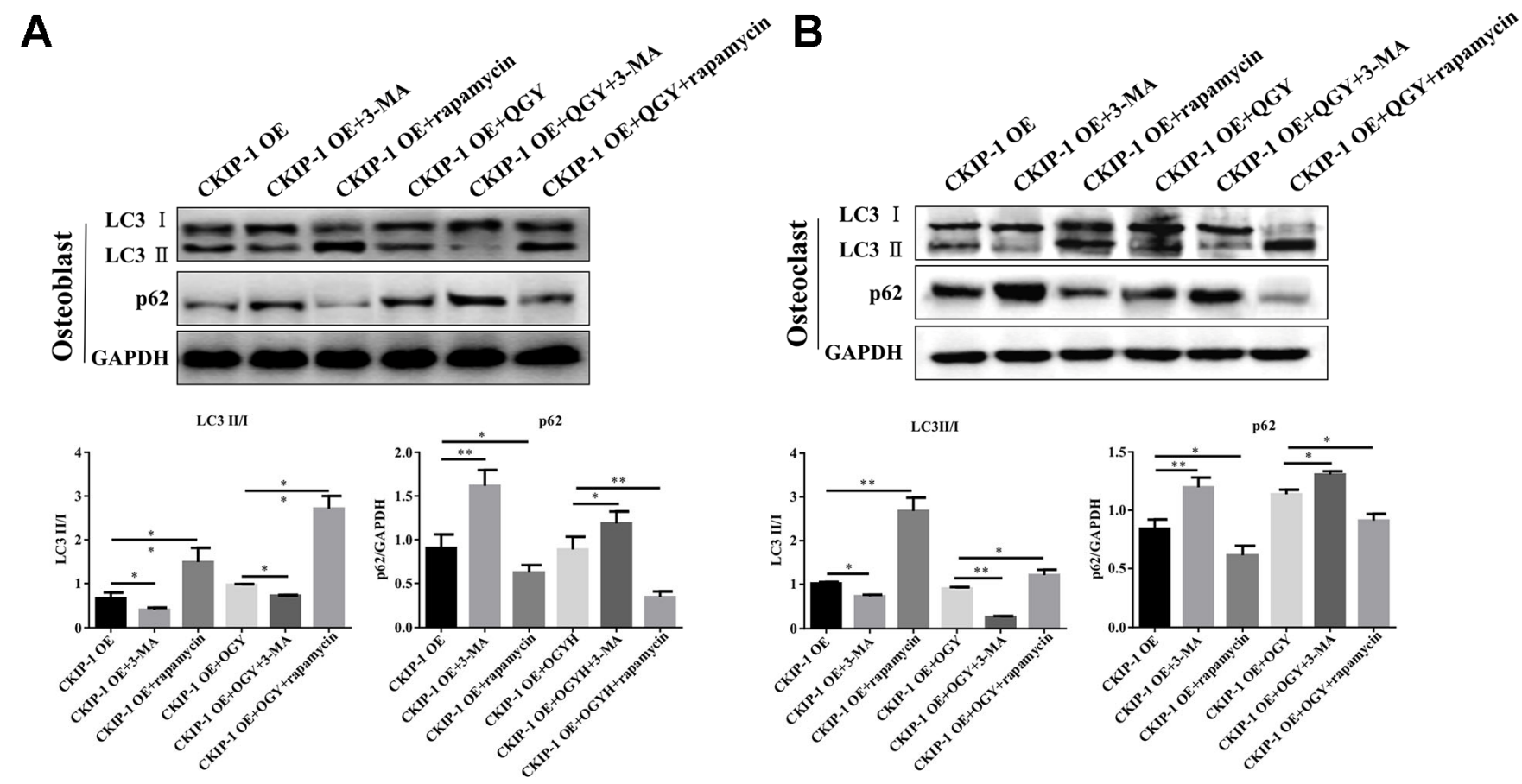

Figure 5. QGY mediates the effect of CKIP-1 on autophagy of osteoblasts and osteoclasts. (A, B) Representative figures and quantitative analysis of protein expression of LC3II/I and p62 in the osteoblasts (A) and osteoclasts (B). The data are presented as the means $\pm \mathrm{SD}(\mathrm{n}=6) .{ }^{*} p<0.05,{ }^{* *} p<0.01$. One-way ANOVA followed by Tukey's post hoc test. 
model of osteoporosis [24]. In another study using a rat mandibular distraction osteogenesis (DO) model, CKIP1 silencing inhibited BMSC apoptosis and promoted osteogenic differentiation [25]. Consistent with previous studies, our research shows that CKIP-1 mediates the autophagy activation of osteoblasts and inhibits differentiation, increases the expression ratio of RANKL/OPG in osteoblasts, and then inhibits cell osteogenesis; CKIP-1 also mediates the autophagy inhibition of osteoclast. Importantly, QGY treatment promotes osteoblast differentiation and enhances osteogenic capacity by targeting CKIP-1 mediated AKT/mTOR pathway activation and autophagy inhibition (rather than osteoclasts), thereby playing a role in improving osteoporosis (Figure 7).

The AKT/mTOR pathway is a key regulator of bone formation [26]. Previous studies have shown that AKT knockout mice have delayed ossification [27]. AKT is also a key factor in the differentiation process of
A

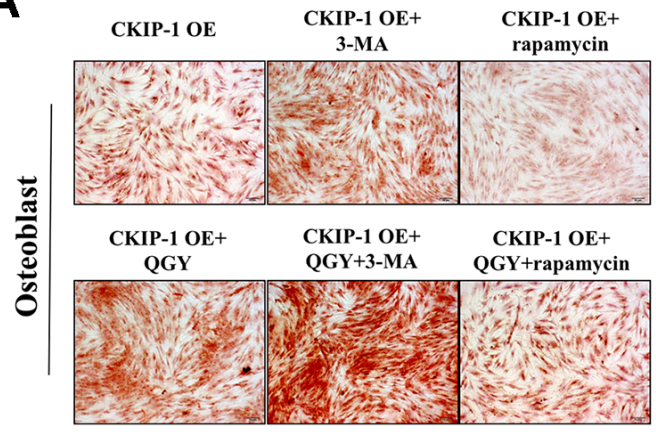

B

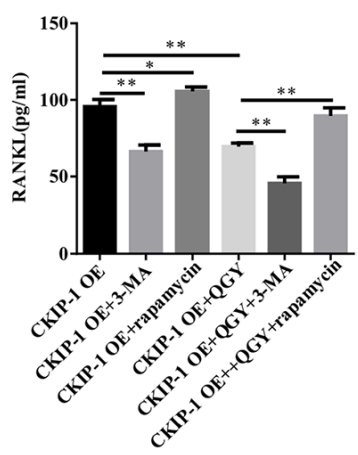

RUNX2
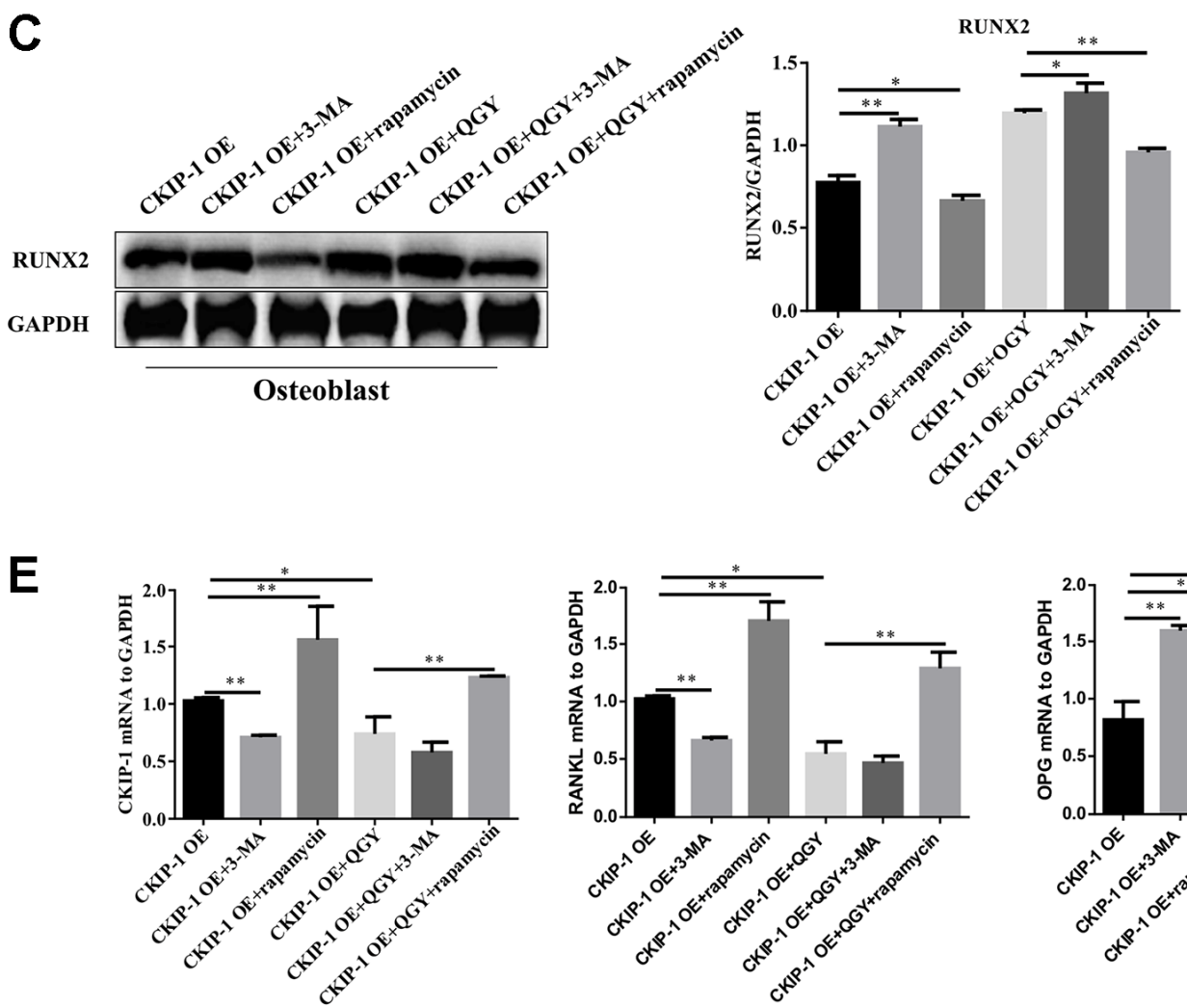

Osteoblast
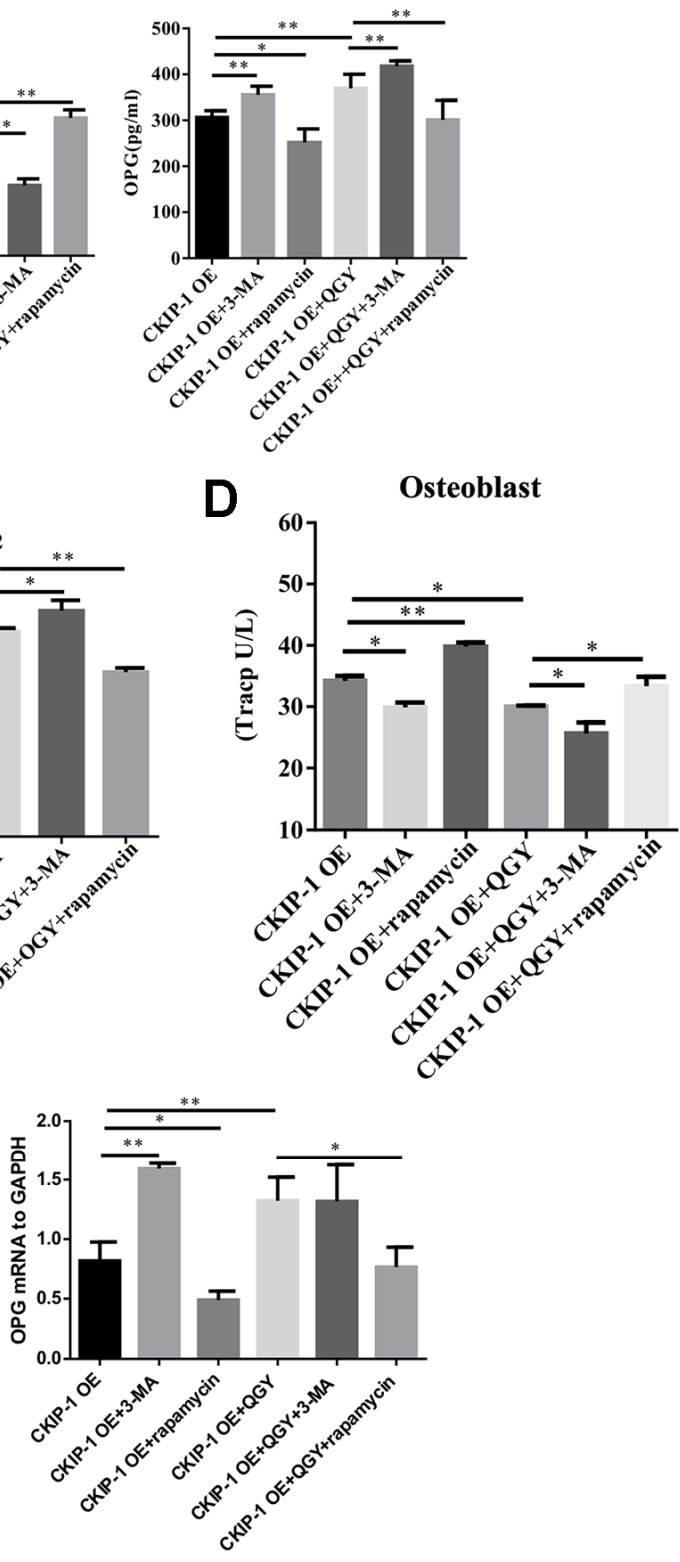

Figure 6. CKIP-1 mediated autophagy on the differentiation and function. (A) Alizarin Red Staining in the osteoblasts. (B) RANKL and OPG content analysis in the osteoblasts. (C) Representative figures and quantitative analysis of protein expression of RUNX2 in the osteoblasts. (D) TRACP activity analysis in the osteoblasts. (E) Gene expression levels of CKIP-1, RANKL and OPG in the osteoblasts. The data are presented as the means \pm S.D $(n=6) .{ }^{*} p<0.05,{ }^{* *} p<0.01$. One-way ANOVA followed by Tukey's post hoc test. 
osteoblasts [28]. Xi et al. reported that the AKT/mTOR signaling pathway participates in the inhibition of osteoporosis by promoting osteoblast proliferation, differentiation and bone formation in vitro and in vivo models [29]. In this study, we found that overexpression of CKIP-1 significantly inhibits the AKT/mTOR pathway, while OGY treatment reactivates the inhibited AKT/mTOR pathway, which is consistent with previous studies [9, 30-35]. Therefore, CKIP-1 acts as an intermediary between QGY and AKT/mTOR signaling crosstalk. After treating osteoblasts with AKT/mTOR pathway inhibitors, it is further proved that QGY exerts anti-osteoporosis effects through AKT/mTOR pathway. In addition, the AKT/mTOR signaling pathway is also one of the most important ways to regulate autophagy. The autophagy pathway is considered to play a supporting role in the development of osteoporosis [3639]. In this study, autophagy was activated during osteoblast differentiation. Among them, ATG5 and p62 have been shown to regulate autophagy degradation [40], and the lipidated form of LC3 converted from LC3-I to LC3-II is considered as an autophagosome marker because it locates and aggregates on autophagosomes [41]. QGY treatment targets CKIP-1 mediated AKT/mTOR pathway activation and autophagy inhibition.

RANKL (receptor activator of nuclear factor kappa B ligand) is a key osteoclast differentiation factor, which is secreted in large quantities by fibroblasts, osteoblasts and stromal cells around the prosthesis. It combines RANK and cooperates with NF- $\kappa \mathrm{B}$, promote osteoclast differentiation and survival, and osteoclast bone resorption; OPG, as an osteoclast differentiation inhibitor, usually competes with RANK to integrate RANKL, blocking the promotion effect of RANKL/RANK on osteoclast and osteoclast bone resorption, thereby inhibiting osteolysis [42]. The imbalance between osteoblast bone formation and osteoclast bone resorption ultimately leads to osteolysis around the prosthesis, where the OPG/RANKL ratio is one of the best characterizing biomarkers related to the pathology of bone destruction [43]. As expected, our results indicate that QGY treatment also promotes osteoblast differentiation and enhances osteogenic capacity, thereby playing a role in improving osteoporosis.

Our data is also supported by other reports. For example, NUPR1 is considered to be a new type of bone formation regulator, and its deficiency will reduce the production of early osteoclasts and enhance the production of osteoblasts, resulting in increased bone formation [44]. In addition, resveratrol regulates the expression of autophagy-related proteins in a dosedependent manner, such as p62, LC3-II, Atg5, Atg7 and Atg12, etc., inhibits autophagy in osteoblasts and activates osteoclast autophagy, and regulates autophagy.

\section{QiangGuYin}

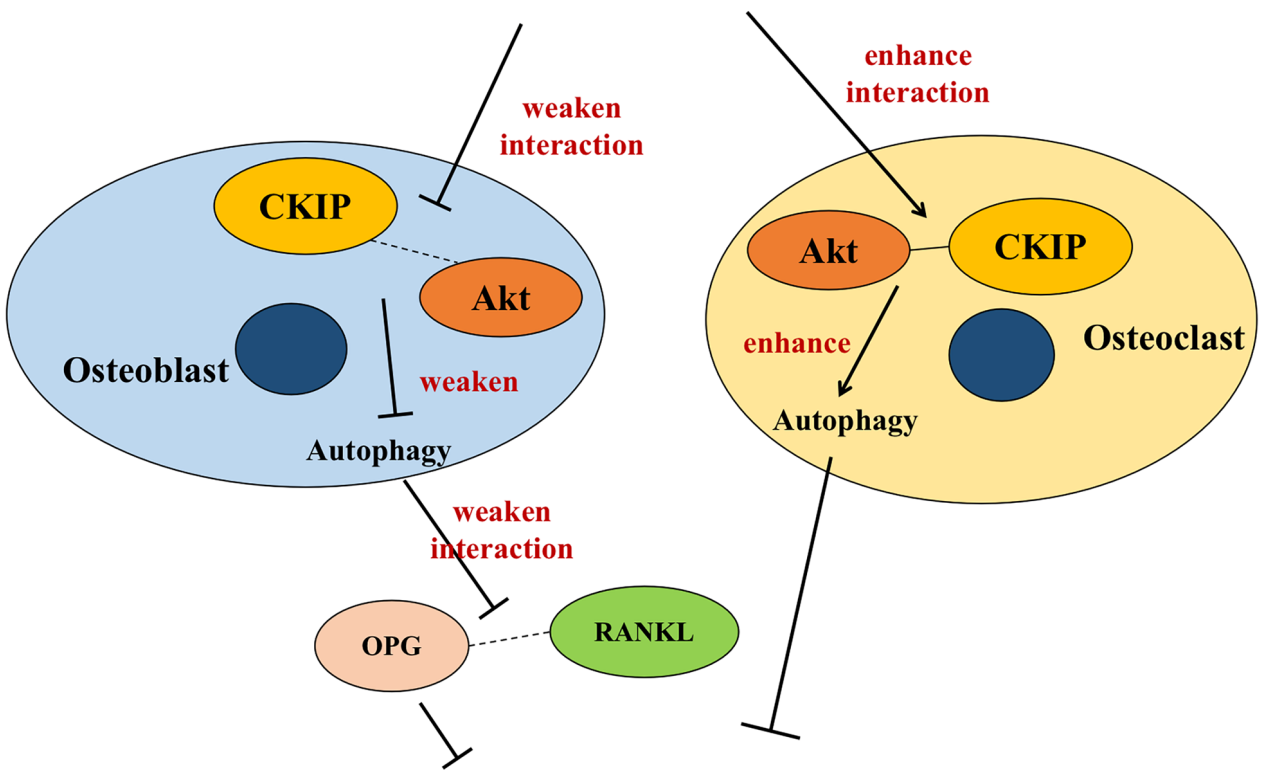

Osteoporosis

Figure 7. Schematic diagram of QGY differential regulation of CKIP-1/AKT/mTOR/autophagy pathways in osteoblasts and osteoclasts. 
It promotes osteoblast differentiation and inhibits osteoclast differentiation, thereby improving postmenopausal osteoporosis [45]. Consistently, our study also showed the opposite autophagy expression and osteogenic ability in osteoblasts and osteoclasts, which may be related to the restoration of the balance between bone formation and bone resorption by QGY regulating the $\mathrm{AKT} / \mathrm{mTOR}$ signaling pathway mediated by CKIP-1.

To sum up, consistent with our previously published clinical trial [20], this study provides new information that QGY treatment promotes growth by targeting CKIP-1 mediated AKT/mTOR pathway activation and autophagy inhibition. Osteocytes differentiate and enhance the ability of bone formation, thereby playing a role in improving osteoporosis. Importantly, activation of autophagy with rapamycin not only reversed the inhibition of QGY on the RANKL/OPG ratio and osteoclast differentiation, but also further enhanced this inhibition by using 3-MA to inhibit autophagy. The synergistic in vivo anti-osteoporosis effect of QGY and 3-MA confirms the results we found in cell research. As far as we know, the current research reveals for the first time the CKIP-1 regulatory mechanism of this popular herbal formula to treat osteoporosis.

\section{AUTHOR CONTRIBUTIONS}

LK and XS designed the study. YY, HZ, SW, JS, CH, TC, MF, SL, SK and HX performed the experiments. BT, BL, YM and JL analyzed data. YY, HZ, SW, JS and $\mathrm{CH}$ wrote the manuscript. All authors read and approved the final manuscript.

\section{CONFLICTS OF INTEREST}

The authors declare that they have no conflicts of interest.

\section{FUNDING}

The study was supported by grant from the National Natural Science Foundation of China [No. 81873129; 81803902; 81973884; 82074183] and Zhejiang Provincial Natural Science Foundation of China [No. LY19H290004].

\section{Editorial Note}

${ }^{\&}$ This corresponding author has a verified history of publications using the personal email address for correspondence.

\section{REFERENCES}

1. Shao B, Liao L, Yu Y, Shuai $Y$, Su X, Jing $H$, Yang D, Jin $Y$. Estrogen preserves Fas ligand levels by inhibiting microRNA-181a in bone marrow-derived mesenchymal stem cells to maintain bone remodeling balance. FASEB J. 2015; 29:3935-44. https://doi.org/10.1096/fj.15-272823 PMID:26062603

2. Ji L, Gao J, Kong R, Gao Y, Ji X, Zhao D. Autophagy exerts pivotal roles in regulatory effects of $1 \alpha, 25$ $(\mathrm{OH})_{2} \mathrm{D}_{3}$ on the osteoclastogenesis. Biochem Biophys Res Commun. 2019; 511:869-74. https://doi.org/10.1016/j.bbrc.2019.02.114 PMID:30850163

3. Basudan $A M$, Shaheen MY, de Vries RB, van den Beucken JJ, Jansen JA, Alghamdi HS. Antiosteoporotic Drugs to Promote Bone Regeneration Related to Titanium Implants: A Systematic Review and MetaAnalysis. Tissue Eng Part B Rev. 2019; 25:89-99. https://doi.org/10.1089/ten.TEB.2018.0120 PMID:30191772

4. Nollet M, Santucci-Darmanin S, Breuil V, Al-Sahlanee R, Cros C, Topi M, Momier D, Samson M, Pagnotta S, Cailleteau L, Battaglia S, Farlay D, Dacquin R, et al. Autophagy in osteoblasts is involved in mineralization and bone homeostasis. Autophagy. 2014; 10:1965-77. https://doi.org/10.4161/auto.36182 PMID:25484092

5. Zhu Y, Zhou J, Ao R, Yu B. A-769662 protects osteoblasts from hydrogen dioxide-induced apoptosis through activating of AMP-activated protein kinase (AMPK). Int J Mol Sci. 2014; 15:11190-203. https://doi.org/10.3390/ijms150611190 PMID:24960362

6. Tarantino U, Iolascon G, Cianferotti L, Masi L, Marcucci G, Giusti F, Marini F, Parri S, Feola M, Rao C, Piccirilli E, Zanetti EB, Cittadini N, et al. Clinical guidelines for the prevention and treatment of osteoporosis: summary statements and recommendations from the Italian Society for Orthopaedics and Traumatology. J Orthop Traumatol. 2017 (Suppl 1); 18:3-36. https://doi.org/10.1007/s10195-017-0474-7 PMID:29058226

7. Yamashita $M$, Ying SX, Zhang GM, Li C, Cheng SY, Deng $C X$, Zhang YE. Ubiquitin ligase Smurf1 controls osteoblast activity and bone homeostasis by targeting MEKK2 for degradation. Cell. 2005; 121:101-13.

https://doi.org/10.1016/i.cell.2005.01.035 PMID:15820682

8. Lu K, Yin X, Weng T, Xi S, Li L, Xing G, Cheng X, Yang X, Zhang $L$, He F. Targeting WW domains linker of HECTtype ubiquitin ligase Smurf1 for activation by CKIP-1. Nat Cell Biol. 2008; 10:994-1002. 
https://doi.org/10.1038/ncb1760

PMID:18641638

9. Zhang L, Zhou Q, Song W, Wu K, Zhang Y, Zhao Y. DualFunctionalized Graphene Oxide Based siRNA Delivery System for Implant Surface Biomodification with Enhanced Osteogenesis. ACS Appl Mater Interfaces. 2017; 9:34722-35.

https://doi.org/10.1021/acsami.7b12079

PMID:28925678

10. Shapiro IM, Layfield R, Lotz M, Settembre C, Whitehouse C. Boning up on autophagy: the role of autophagy in skeletal biology. Autophagy. 2014; 10:7-19.

https://doi.org/10.4161/auto.26679 PMID:24225636

11. Pierrefite-Carle V, Santucci-Darmanin S, Breuil V, Camuzard O, Carle GF. Autophagy in bone: Self-eating to stay in balance. Ageing Res Rev. 2015; 24:206-17. https://doi.org/10.1016/j.arr.2015.08.004 PMID:26318060

12. Greenhill C. Bone: Autophagy regulates bone growth in mice. Nat Rev Endocrinol. 2016; 12:4.

https://doi.org/10.1038/nrendo.2015.214 PMID:26635109

13. Bo T, Yan F, Guo J, Lin X, Zhang H, Guan Q, Wang H, Fang L, Gao L, Zhao J, Xu C. Characterization of a Relatively Malignant Form of Osteopetrosis Caused by a Novel Mutation in the PLEKHM1 Gene. J Bone Miner Res. 2016; 31:1979-87. https://doi.org/10.1002/jbmr.2885 PMID:27291868

14. Luo D, Li J, Chen K, Rong X, Guo J. Untargeted Metabolomics Reveals the Protective Effect of Fufang Zhenshu Tiaozhi (FTZ) on Aging-Induced Osteoporosis in Mice. Front Pharmacol. 2019; 9:1483. https://doi.org/10.3389/fphar.2018.01483 PMID:30670964

15. Wang L, Zhou GB, Liu P, Song JH, Liang $Y$, Yan XJ, Xu F, Wang BS, Mao JH, Shen ZX, Chen SJ, Chen Z. Dissection of mechanisms of Chinese medicinal formula RealgarIndigo naturalis as an effective treatment for promyelocytic leukemia. Proc Natl Acad Sci USA. 2008; 105:4826-31.

https://doi.org/10.1073/pnas.0712365105 PMID:18344322

16. Wang $Q$, Zhao $Y$, Sha $N$, Zhang Y, Li C, Zhang H, Tang D, Lu S, Shi Q, Wang Y, Shu B, Zhao D. The systemic bone protective effects of Gushukang granules in ovariectomized mice by inhibiting osteoclastogenesis and stimulating osteoblastogenesis. J Pharmacol Sci. 2018; 136:155-64. https://doi.org/10.1016/i.jphs.2018.01.007 PMID:29501580

17. Zhang JL, Qiu XM, Zhang N, Tang W, Gober HJ, Li DJ, Wang L. Bu-Shen-Ning-Xin decoction suppresses osteoclastogenesis by modulating RANKL/OPG imbalance in the CD4+ $T$ lymphocytes of ovariectomized mice. Int J Mol Med. 2018; 42:299-308. https://doi.org/10.3892/ijmm.2018.3645 PMID:29717766

18. Liang BC, Shi XL, Li CW, Shi ZY, He WT, Yao JL, Kong LC, $\mathrm{Li} X Y$. Identification of human serum protein targets of Qianggu Decoction () in primary type I osteoporosis based on tandem mass tag labeling and liquid chromatography-tandem mass spectrometry technology. Chin J Integr Med. 2017; 23:747-54. https://doi.org/10.1007/s11655-016-2600-4 PMID:27389089

19. Jiannong $\mathrm{W}$, Junjie J, Yanming $\mathrm{X}, \mathrm{Xu} \mathrm{W}$, Jianpeng $\mathrm{L}$, Jingli $D$, Xin X. Effect of naringenin in Qianggu capsule on population pharmacokinetics in Chinese women with primary osteoporosis. J Tradit Chin Med. 2015; 35:141-53. https://doi.org/10.1016/s0254-6272(15)30021-2 PMID:25975046

20. Shi ZY, Zhang XG, Li CW, Liu K, Liang BC, Shi XL. Effect of Traditional Chinese Medicine Product, QiangGuYin, on Bone Mineral Density and Bone Turnover in Chinese Postmenopausal Osteoporosis. Evid Based Complement Alternat Med. 2017; 2017:6062707. https://doi.org/10.1155/2017/6062707 PMID:28512501

21. Shi GX, Zheng XF, Zhu C, Li B, Wang YR, Jiang SD, Jiang LS. Evidence of the Role of R-Spondin 1 and Its Receptor Lgr4 in the Transmission of Mechanical Stimuli to Biological Signals for Bone Formation. Int J Mol Sci. 2017; 18:564. https://doi.org/10.3390/ijms18030564 PMID:28272338

22. Liu J, Lu C, Wu X, Zhang Z, Li J, Guo B, Li D, Liang C, Dang L, Pan X, Peng S, Lu A, Zhang B, Zhang G. Targeting osteoblastic casein kinase-2 interacting protein-1 to enhance Smad-dependent BMP signaling and reverse bone formation reduction in glucocorticoid-induced osteoporosis. Sci Rep. 2017; 7:41295. https://doi.org/10.1038/srep41295 PMID:28128304

23. Han B, Wei SP, Zhang XC, Li H, Li Y, Li RX, Li K, Zhang XZ. Effects of constrained dynamic loading, CKIP-1 gene knockout and combination stimulations on bone loss caused by mechanical unloading. Mol Med Rep. 2018; 18:2506-14.

https://doi.org/10.3892/mmr.2018.9222 PMID:29956799

24. Zhang L, Wu K, Song W, Xu H, An R, Zhao L, Liu B, Zhang Y. Chitosan/siCkip-1 biofunctionalized titanium implant for improved osseointegration in the osteoporotic condition. Sci Rep. 2015; 5:10860. 
https://doi.org/10.1038/srep10860 PMID:26040545

25. Zhou ZC, Che L, Kong L, Lei DL, Liu R, Yang XJ. CKIP-1 silencing promotes new bone formation in rat mandibular distraction osteogenesis. Oral Surg Oral Med Oral Pathol Oral Radiol. 2017; 123:e1-e9. https://doi.org/10.1016/j.oooo.2016.07.013 PMID:27727105

26. Guntur AR, Rosen CJ. The skeleton: a multi-functional complex organ: new insights into osteoblasts and their role in bone formation: the central role of PI3Kinase. J Endocrinol. 2011; 211:123-30. https://doi.org/10.1530/JOE-11-0175 PMID:21673026

27. Peng $X D, X u P Z$, Chen $M L$, Hahn-Windgassen $A$, Skeen J, Jacobs J, Sundararajan D, Chen WS, Crawford SE, Coleman KG, Hay N. Dwarfism, impaired skin development, skeletal muscle atrophy, delayed bone development, and impeded adipogenesis in mice lacking Akt1 and Akt2. Genes Dev. 2003; 17:1352-65.

https://doi.org/10.1101/gad.1089403

PMID:12782654

28. Sugatani T, Hruska KA. Akt1/Akt2 and mammalian target of rapamycin/Bim play critical roles in osteoclast differentiation and survival, respectively, whereas Akt is dispensable for cell survival in isolated osteoclast precursors. J Biol Chem. 2005; 280:3583-9.

https://doi.org/10.1074/jbc.M410480200

PMID:15545269

29. Xi JC, Zang HY, Guo LX, Xue HB, Liu XD, Bai YB, Ma YZ. The PI3K/AKT cell signaling pathway is involved in regulation of osteoporosis. J Recept Signal Transduct Res. 2015; 35:640-5.

https://doi.org/10.3109/10799893.2015.1041647 PMID:26390889

30. Zhang L, Wang Y, Xiao F, Wang S, Xing G, Li Y, Yin X, Lu K, Wei R, Fan J, Chen Y, Li T, Xie P, et al. CKIP-1 regulates macrophage proliferation by inhibiting TRAF6-mediated Akt activation. Cell Res. 2014; 24:742-61.

https://doi.org/10.1038/cr.2014.53

PMID:24777252

31. Guo B, Zhang B, Zheng L, Tang T, Liu J, Wu H, Yang Z, Peng S, He X, Zhang H, Yue KK, He F, Zhang L, et al. Therapeutic RNA interference targeting CKIP-1 with a cross-species sequence to stimulate bone formation. Bone. 2014; 59:76-88.

https://doi.org/10.1016/j.bone.2013.11.007

PMID:24246247

32. Fu L, Zhang L. Physiological functions of CKIP-1: From molecular mechanisms to therapy implications. Ageing Res Rev. 2019; 53:100908. https://doi.org/10.1016/j.arr.2019.05.002 PMID:31082489

33. Li L, Xie P, Lin W, Liu J, Chen J, Guo Z, Bin C, An W, Zhang C, Zhan Y. CKIP-1 augments autophagy in steatotic hepatocytes by inhibiting Akt/mTOR signal pathway. Exp Cell Res. 2020; 397:112341. https://doi.org/10.1016/j.yexcr.2020.112341 PMID:33191205

34. Liu Q, Guo Y, Wang Y, Zou X, Yan Z. miR-98-5p promotes osteoblast differentiation in MC3T3-E1 cells by targeting CKIP-1. Mol Med Rep. 2018; 17:4797-802. https://doi.org/10.3892/mmr.2018.8416 PMID:29328483

35. Peng X, Wu X, Zhang J, Zhang G, Li G, Pan X. The role of CKIP-1 in osteoporosis development and treatment. Bone Joint Res. 2018; 7:173-8.

https://doi.org/10.1302/2046-3758.72.BJR-20170172.R1 PMID:29682283

36. Toscani D, Bolzoni M, Ferretti M, Palumbo C, Giuliani N. Role of Osteocytes in Myeloma Bone Disease: Antisclerostin Antibody as New Therapeutic Strategy. Front Immunol. 2018; 9:2467.

https://doi.org/10.3389/fimmu.2018.02467 PMID:30410490

37. Luo D, Ren H, Li T, Lian K, Lin D. Rapamycin reduces severity of senile osteoporosis by activating osteocyte autophagy. Osteoporos Int. 2016; 27:1093-101. https://doi.org/10.1007/s00198-015-3325-5 PMID:26395886

38. Yao Z, Lei W, Duan R, Li Y, Luo L, Boyce BF. RANKL cytokine enhances TNF-induced osteoclastogenesis independently of TNF receptor associated factor (TRAF) 6 by degrading TRAF3 in osteoclast precursors. J Biol Chem. 2017; 292:10169-79. https://doi.org/10.1074/jbc.M116.771816 PMID:28438834

39. Xu X, Wang R, Wu R, Yan W, Shi T, Jiang Q, Shi D. Trehalose reduces bone loss in experimental biliary cirrhosis rats via ERK phosphorylation regulation by enhancing autophagosome formation. FASEB J. 2020; 34:8402-15.

https://doi.org/10.1096/fj.201902528RRR PMID:32367591

40. Aoki S, Shimizu K, Ito K. Autophagy-dependent mitochondrial function regulates osteoclast differentiation and maturation. Biochem Biophys Res Commun. 2020; 527:874-80.

https://doi.org/10.1016/j.bbrc.2020.04.155 PMID: $\underline{32430180}$

41. Fu L, Wu W, Sun X, Zhang P. Glucocorticoids Enhanced Osteoclast Autophagy Through the PI3K/Akt/mTOR Signaling Pathway. Calcif Tissue Int. 2020; 107:60-71. 
https://doi.org/10.1007/s00223-020-00687-2

PMID:32274533

42. Zhu F, Wang J, Ni Y, Yin W, Hou Q, Zhang Y, Yan S, Quan R. Curculigoside Protects against Titanium ParticleInduced Osteolysis through the Enhancement of Osteoblast Differentiation and Reduction of Osteoclast Formation. J Immunol Res. 2021; 2021:5707242.

https://doi.org/10.1155/2021/5707242

PMID:34285923

43. Xie L, Xie H, Chen C, Tao Z, Zhang C, Cai L. Inhibiting the $\mathrm{PI}$ IK/AKT/NF-KB signal pathway with nobiletin for attenuating the development of osteoarthritis: in vitro and in vivo studies. Food Funct. 2019; 10:2161-75.

https://doi.org/10.1039/c8fo01786g

PMID: $\underline{0938722}$

44. Shiraki $M, X u X$, lovanna JL, Kukita $T$, Hirata $H$, Kamohara A, Kubota $Y$, Miyamoto $H$, Mawatari $M$, Kukita A. Deficiency of stress-associated gene Nupr1 increases bone volume by attenuating differentiation of osteoclasts and enhancing differentiation of osteoblasts. FASEB J. 2019; 33:8836-52.

https://doi.org/10.1096/fj.201802322RR

PMID:31067083

45. Wang W, Zhang LM, Guo C, Han JF. Resveratrol promotes osteoblastic differentiation in a rat model of postmenopausal osteoporosis by regulating autophagy. Nutr Metab (Lond). 2020; 17:29.

https://doi.org/10.1186/s12986-020-00449-9

PMID: $\underline{32322287}$ 